\title{
Microwave Imaging Reflectometry System for KSTAR ${ }^{*}$
}

\author{
Woochang LEE, Gunsu S. YUN, Inho HONG, Minwoo KIM, Jincheol B. KIM, \\ Yoonbum NAM, Hyeon K. PARK, Young G. KIM ${ }^{1)}$, Kang W. KIM ${ }^{1)}$, Benjamin TOBIAS ${ }^{2)}$, \\ Calvin W. DOMIER ${ }^{2)}$ and Neville C. LUHMANN, Jr. ${ }^{2)}$ \\ POSTECH, Pohang, Gyeongbuk 790-784, Korea \\ 1) Kyungpook National University, Daegu 702-701, Korea \\ ${ }^{2)}$ University of California at Davis, California 95616, USA
}

(Received 5 December 2010 / Accepted 18 March 2011)

\begin{abstract}
A new microwave imaging reflectometry (MIR) system for KSTAR is being developed based on the experience gained via the TEXTOR proof-of-principle system [H. Park et al., Rev. Sci. Instrum. 74, 4239 (2003)] which aimed to measure the poloidal image of the electron density fluctuations essential for transport studies. The KSTAR system will adopt a multi-frequency probe beam source in the range of $90 \sim 100 \mathrm{GHz}$ (X-mode case), which will enable the measurement of 2-D (radial and poloidal) fluctuations of the multiple cut-off layers, simultaneously. The optical system of the MIR system will be combined with the 2nd ECEI system (identical to the first ECEI system [G.S. Yun et al., Rev. Sci. Instrum. 81, 10D930 (2010)]) on KSTAR. The design of the launching and receiving optics of the MIR system will be constrained in order to maintain the performance of the ECEI system and thus it is necessary to consider sharing the zoom lens of the ECEI system. This stringent constraint is a challenge considering the tight wavefront matching requirement to obtain proper images for a wide range of cut-off layers within the focal depth. This paper discusses the details of the MIR system design that is compatible with the 2nd ECEI system on KSTAR.
\end{abstract}

(C) 2011 The Japan Society of Plasma Science and Nuclear Fusion Research

Keywords: microwave imaging reflectometry, two dimensional image, electron density fluctuation

DOI: $10.1585 /$ pfr.6.2402037

\section{Introduction and Background}

Microwave reflectometry has been widely employed for a number of decades in measuring the electron density profile and density fluctuations in a magnetically confined plasma. It has advantages such as straightforward operating principle similar to the radar, relatively simple implementation, and its high sensitivity to small density fluctuations near the cut-off layer. However, interpretation of the reflectometry data from density fluctuations is still an important issue since the reflected wave from the reflecting layer forms a complicated interference pattern at the detector plane in the presence of strong density fluctuations and high wavenumbers [1-4].

The basic principle of microwave reflectometry is that the microwave beam with a frequency $\omega$ is launched into the plasma along the density gradient and is reflected at the layer where the electron density equals a critical value [5]. This reflecting layer is referred to as a cut-off layer. Phase changes in the reflected beam are measured by mixing this beam with a reference beam in a detector. The electron density profile is measured by determining the relative positions of the different density layers with a range of different probing frequencies [6]. On the other hand, density

author's e-mail: woochanglee @ postech.ac.kr

*) This article is based on the presentation at the 20th International Toki Conference (ITC20). fluctuations are detected by measuring the phase movement of the reflected beam with a constant frequency probe beam.

In a conventional microwave reflectometry, the probing wave is launched and received on the equatorial plane using a pair of small horn antennas. The measurement is essentially a point measurement, and does not provide direct information on the spatial structure of the fluctuations [7]. Using correlation technique, the radial structure of plasma fluctuations can be deduced from the reflected waves by closely spaced cut-off layers.

The 1D geometrical optics approximation breaks down in the case of multidimensional turbulent fluctuations, which exhibit both radial and poloidal variations $[3,4,7]$. In this case, under the condition of sufficiently large amplitude and/or wave number turbulence, the reflected field at the detector plane will be complicated due to the interference of the wave fronts. Thus, it would be difficult to extract any accurate information of the density fluctuations at the cut-off layer. Number of techniques have been employed to account for the interference effect of 2D fluctuation. As an example, the reflectometer signals are adjusted numerically by back projecting the measured complex electric fields through the modeled dispersive plasma medium [8].

Studies of this problem, both on the Tokamak Fusion 


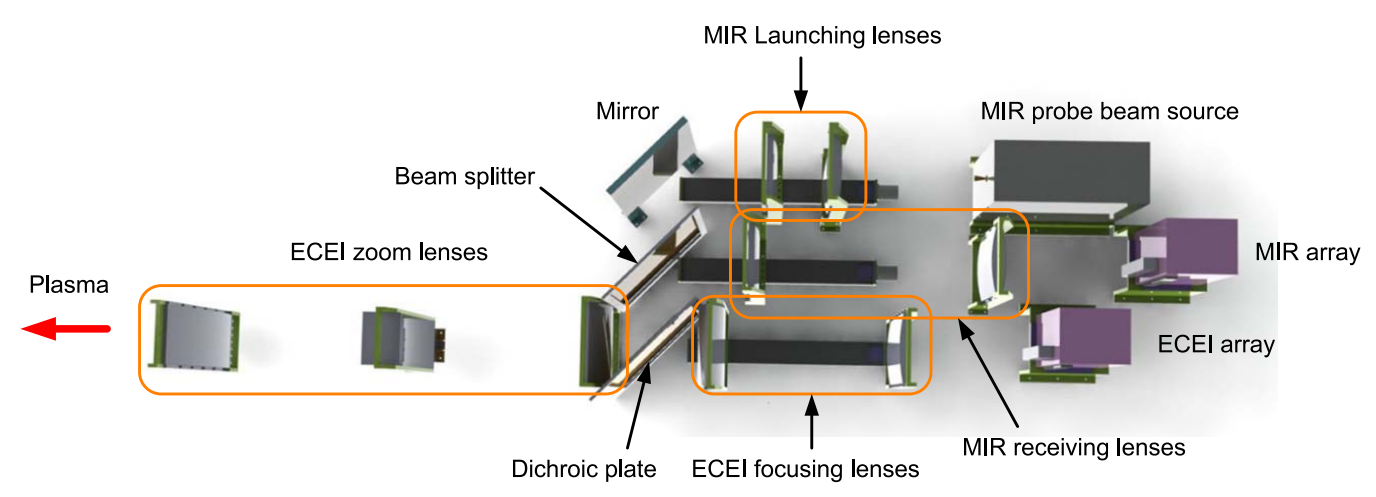

Fig. 1 Conceptual design of the combined MIR and 2nd ECEI system on KSTAR. The dichroic plate is used to separate the MIR and ECEI signals.

Test Reactor (TFTR) and in a series of numerical simulation, led to the development of the microwave imaging reflectometry (MIR) concept [9]. In this technique, largeaperture optics at the plasma edge is used to shine the probe beam on a wide region of the cut-off layer, and collect back as much of the reflected beam as possible, and form an image of the cut-off layer onto the 1D array of detectors. The first MIR instrument of this type was installed on the TEXTOR tokamak [3,4]. The TEXTOR MIR system with a fixed frequency source of $88 \mathrm{GHz}$ and linear array of detectors consisting of 16 elements, covers $\lesssim 15 \mathrm{~cm}$ poloidal region of the cut-off surface with a spatial resolution of $\sim 1 \mathrm{~cm}$, leading to a $k_{\theta}$ resolution of $0.4 \mathrm{~cm}^{-1} \leqslant k_{\theta} \leqslant 3 \mathrm{~cm}^{-1}$. Laboratory test of the TEXTOR system using a corrugated metal reflector attached to the wheel with a matching curvature demonstrated the advantage of MIR system over the conventional reflectometry. However, the test result from the plasma cut-off layer did not meet the expectation of the MIR system. Extensive test of the prototype was performed at POSTECH and found fatal mistakes [10].

\section{KSTAR MIR System}

The KSTAR MIR system is being developed to measure 2-dimensional (radial $\times$ poloidal) density fluctuations for turbulence study. The radial measurement can be performed by multi-frequency probe beam sources since probe beams in different frequencies reflect from different cut-off layers, and the poloidal measurement can be done by illuminating the probe beam into the wide region of the cut-off layer within the focal depth. A full system, that is planned to be installed by 2014, will have 5 radial and 20 poloidal detection channels. The target plasma parameter is $B_{\mathrm{t}}=3.0 \sim 3.5 \mathrm{~T}, n_{\mathrm{e}}=2 \sim 5 \times 10^{19} \mathrm{~m}^{-3}$, elongation $=1.6 \sim 1.8$, and triangularity $\sim 0.6$. Prior to the full MIR system, we are planning to install a prototype MIR system, that has 2 radial and 10 poloidal channels by 2012 KSTAR campaign. The MIR system will share a part of optics, referred as zoom optics, with the planned 2nd ECEI system which is identical to the first system [11]. The two system will simultaneously measure the electron tempera-
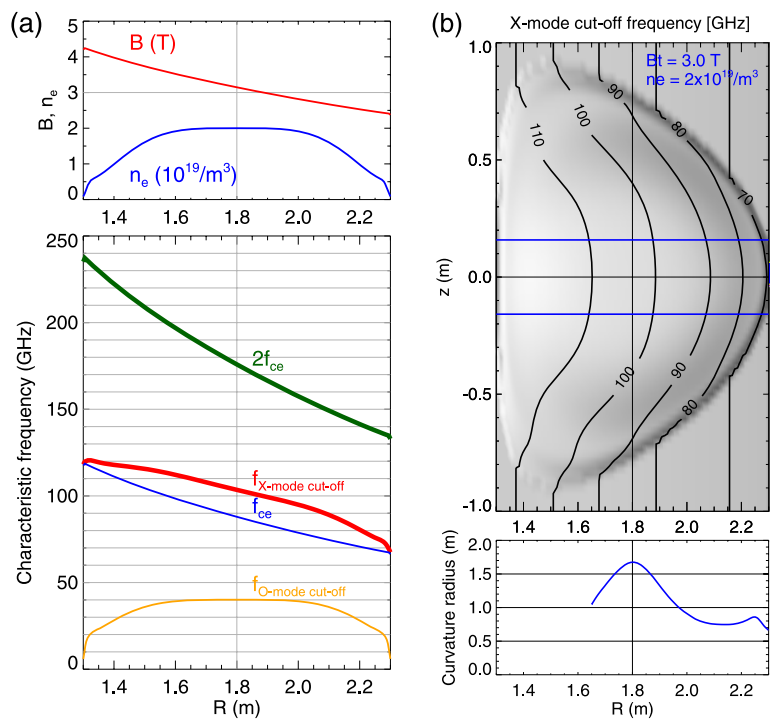

Fig. 2 (a) Frequency range of the KSTAR MIR (red colored Xmode cut-off frequency) and ECEI (green colored 2nd harmonic electron cyclotron frequency, $2 f_{\text {ce }}$ ) system. (b) Contour of the X-mode cut-off frequency (upper) and the radius of curvature of the cut-off layers (lower).

ture fluctuation and density fluctuation in the outboard region of the KSTAR plasma. Figure 1 shows the combined KSTAR MIR and ECEI system and Figure 2 (a) shows the frequency ranges of the MIR $(70 \sim 120 \mathrm{GHz})$ and ECEI system $(135 \sim 235 \mathrm{GHz})$, which are completely seperated.

\subsection{Optics design}

There are a few critical points in the design of the launching and receiving optics of the MIR system. The design of the launching optics has to consider the matching condition of the curvature of the probe beam wavefront with that of the plasma cut-off layer for the optical robustness, and shine the probe beam on a wide region of the cut-off layer, within the optical depth, to get the spatial structure of the fluctuations. The receiving optics should restore phase front image of the reflecting layer (cut-off layer) on the detector plane by collecting as much of the reflected beam as possible using large aperture optics. Al- 


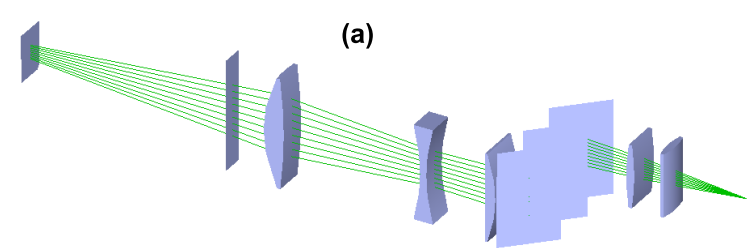

(b)

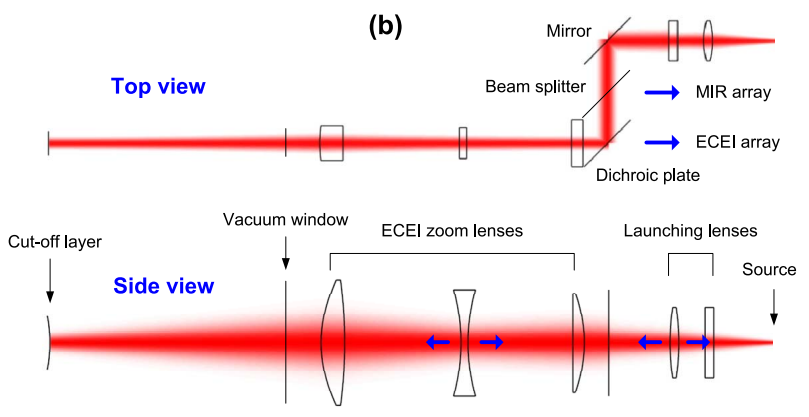

Fig. 3 Launching optics of the KSTAR MIR system: (a) ray trace and (b) Gaussian beam trace. The remotely controllable lenses (concave zoom lens and E-plane launching lens) can adjust the curvature radius of the probe beam wavefront and radial position of the cut-off layer, which are affected by the plasma parameter.

though a tall probe beam is required, one should consider the diffraction effect which deteriorates the phase reconstruction especially in the edge channels. Generally, the aperture size is limited by a port size and a proper probe beam size should be determined based on the aperture size and diffraction analysis.

Figure 3 shows the design of the MIR launching optics. The wavefront of the probing Gaussian beam is matched with the target cut-off layer poloidally and toroidally. The remotely controllable lenses such as 2 nd concave zoom lens and E-plane launching lens, can adjust the curvature radius of the probe beam and focal position based on the plasma parameters. The beam size is largest at the first zoom lens and will be kept below one half of the lens height. The probe beam covers $\sim 11 \mathrm{~cm}$ poloidal region of the cut-off surface with spatial resolution of of $\sim 1 \mathrm{~cm}$, determined by the 10 poloidal channel, so that the detectable wave number of density fluctuation is $\lesssim 1.5 \mathrm{~cm}^{-1}$. Figure 4 shows a limitation of the designed launching optics in the fluctuation wave number and amplitude. The vertical radius is the reflected beam radius, defined as $1 / e^{2}$ intensity, at the first zoom lens from various fluctuating plasma cut-off layers, which are represented by sinusoidal corrugations with three different wave numbers $\left(1.26,0.63\right.$, and $\left.0.42 \mathrm{~cm}^{-1}\right)$ and four different corrugation depths $(0.83,1.7,3.3$, and $6.6 \mathrm{~mm})$. The corrugation depths, peak-to-peak depth of the corrugated surface, are equivalent to $\delta n_{\mathrm{e}} / n_{\mathrm{e}} \sim 0.3,0.6,1.2$, and $2.4 \%$ near $r / a=0.5$ in the $3 \mathrm{~T}$ plasma. The reflected beam radius should be less than the vertical radius of the first zoom lens, which is marked with the thick gray line. The simulation result tells us that fluctuations with higher wave number (or shorter wavelength) and larger amplitude will not be

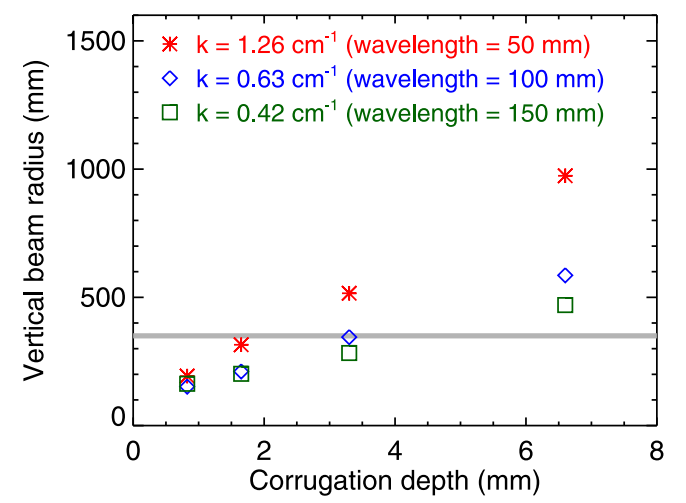

Fig. 4 The limitation in the fluctuating wave number and amplitude was simulated for the optics design. The vertical radius of the reflected beam at the first zoom lens should be shorter than the lens size presented with the gray line.

coupled to the detector array and the images of the cut-off layers can not be reconstructed. The design of the receiving optics is quite delicate and the criteria is being studied experimentally and simulatively.

\subsection{Hardware design}

A prototype MIR system will adopt two-frequency ( 88 and $92 \mathrm{GHz}$ center frequency) millimeter wave source for measuring two separate cut-off layers, and the sources have frequency tunability of $\pm 1 \mathrm{GHz}$ so that we can adjust the cut-off layer slightly based on the plasma parameter. The 88 and $92 \mathrm{GHz}$ probe beam reflect near $r / a \sim 0.5$ in the $3 \mathrm{~T}$ plasma (see Figure 2(b)) and $r / a \sim 0.8$ in the $3.5 \mathrm{~T}$ plasma. The two frequency waves are combined in a diplexer and are launched toward the plasma through a single launching horn antenna. The final output power is $\sim 80 \mathrm{~mW}$ in each frequency.

Two reflected waves from the plasma cut-off layers are coupled to the 10-channel detector array by the receiving optics. The main difference of the KSTAR MIR system compared to the TEXTOR MIR system is the local oscillator (LO) wave coupling method. For the TEXTOR MIR system, the high frequency $(\sim 88 \mathrm{GHz})$ LO signal was optically coupled to the detector array. On the other hand, the KSTAR system will employ a low frequency $(\sim 15 \mathrm{GHz})$ synthesizer as the LO source (see Figure 5). The low frequency LO power is evenly divided into 10 and directly provided into the detector. The detector, 7 th harmonic mixer, downconverts the reflected wave signals from $\sim 90 \mathrm{GHz}$ to the intermediate frequency (IF) $\sim 1$ and $\sim 3 \mathrm{GHz}$. The 1 and $3 \mathrm{GHz}$ reference IF signals drive tracing circuits that generate constant high power reference IF signals. The high power reference IF signal is split into 10 signals and they are transfered to the next mixing stage. The reflected IF signal is mixed with the reference IF signal by an inphase-quadrature (I/Q) demodulator and downconverted to the plasma fluctuation frequency. The I/Q signals are amplified by video amplifiers and recorded by a digitizer with $2 \sim 4 \mathrm{MS} / \mathrm{s}$ sampling rate and 14 bit resolution. 


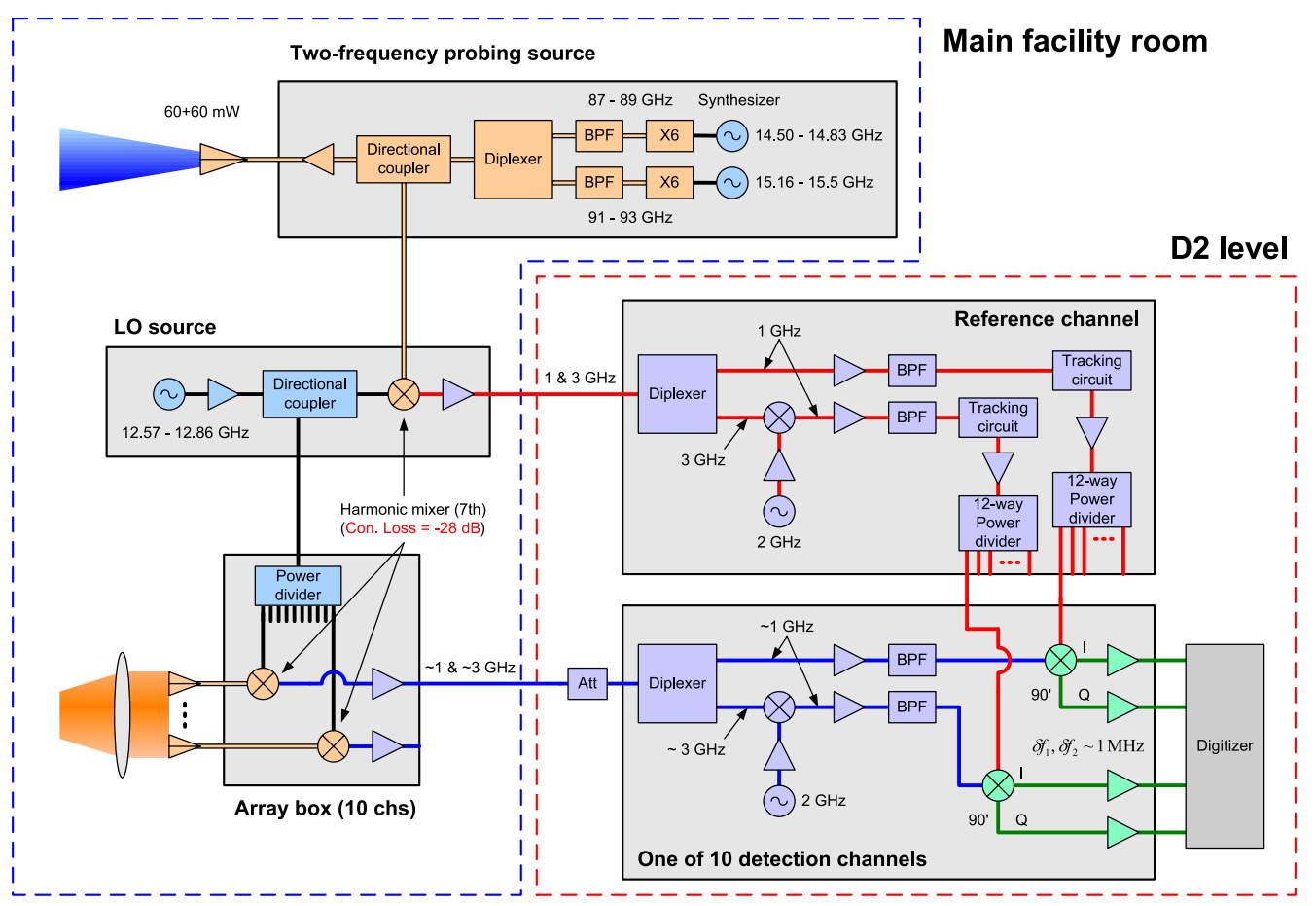

Fig. 5 Schematic of the prototype KSTAR MIR system. The system is composed of two-frequency probe beam source, local oscillator (LO) source, 10-channel detector array, and electronics.

\section{Summary}

The MIR system is being developed on KSTAR to measure two dimensional image of electron density fluctuation with 2 radial and 10 poloidal channels. The full system with 5 radial and 20 poloidal channels will be installed by 2014. The launching and receiving optics of the MIR system is designed to use the zoom lenses of 2nd ECEI system due to the limited port access on KSTAR. The remotely controlled launching optics provides a wide coverage of the curvature radius ranging from 450 to $1000 \mathrm{~mm}$. The fluctuation wave number can be detectable up to $1.5 \mathrm{~cm}^{-1}$ but this is further limited as the fluctuating amplitude increases. The design of the receiving optics is quite difficult so that further studies are required experimentally and simulatively.

The hardware of the MIR system has been designed to implement two-frequency probe beam and 10 channel detector array. The two frequency proving waves combine by a diplexer and launched toward plasma by a single horn antenna. The reflected waves are coupled into vertically aligned 10 channel detector array by the receiving optics and mixed with evenly distributed LO signals. The detail components will be determined and the noise analysis of the whole system will be performed.

\section{Acknowledgments}

This work was supported by NRF of Korea under contract No. 20100020034 and the U.S. DoE under contract No. DE-FG03-95ER-54295.
[1] E. Mazzucato and R. Nazikian, Phys. Rev. Lett. 71, 1840 (1993).

[2] R. Nazikian and E. Mazzucato, Rev. Sci. Instrum. 66, 392 (1995).

[3] T. Munsat, E. Mazzucato, H. Park, C.W. Domier, N.C. Luhmann, Jr., A.J.H. Donné and M. van de Pol, Plasma Phys. Control. Fusion 45, 469 (2003).

[4] H. Park, T. Munsat, E. Mazzucato, C.W. Domier, M. Johnson, N.C. Luhmann, Jr., J. Wang, Z.G. Xia, I.G.J. Classen, A.J.H. Donné and M. van de Pol, Rev. Sci. Instrum. 75, 3787 (2004).

[5] J. Wesson, Tokamaks (Clarendon Press, Oxford, 2004) p. 511.

[6] C. Laviron, A.J.H. Donné, M.E. Manso and J. Sanchez, Plasma Phys. Control. Fusion 38, 905 (1996).

[7] H. Park, C.C. Chang, B.H. Deng, C.W. Domier, A.J.H. Donné, K. Kawahata, C. Liang, X.P. Liang, H.J. Lu, N.C. Luhmann, Jr., A. Mase, H. Matsuura, E. Mazzucato, A. Miura, K. Mizuno, T. Munsat, Y. Nagayama, M.J. van de Pol, J. Wang, Z.G. Xia and W-K. Zhang, Rev. Sci. Instrum. 74, 4239 (2003).

[8] R. Nazikian, J. Mod. Opt. 44, 1037 (1997).

[9] E. Mazzucato, T. Munsat, H. Park, B.H. Deng, C.W. Domier, N.C. Luhmann, Jr., A.J.H. Donné and M.J. van de Pol, Phys. Plasmas 9, 1955 (2002).

[10] H.K. Park, I. Hong, M. Kim, G.S. Yun, W. Lee, J. Kim, B. Tobias, C.W. Domier, N.C. Luhmann, Jr. and K.W. Kim, Rev. Sci. Instrum. 81, 10D933 (2010).

[11] G.S. Yun, W. Lee, M.J. Choi, J.B. Kim, H.K. Park, C.W. Domier, B. Tobias, T. Liang, X. Kong, N.C. Luhmann, Jr. and A.J.H. Donné, Rev. Sci. Instrum. 81, 10D930 (2010). 